\title{
Decrease in sow prolificacy at the second litter : analysis of data from two experimental herds
}

\author{
J. LUCBERT, O. LAVOREL \\ I.T.C.F., 8, avenue du Président-Wilson, 75116 Paris \\ France
}

Today many sow herds kept in intensive management conditions exhibit a reduced prolificacy in the second litter while former results had shown an improvement of prolificacy with the litter order from the first to the fifth cycle. The data collected since 1976 in experimental farms exhibiting this reduction in prolificacy were analysed using the multivariate analysis to point out the factors affecting this phenomenon. Data concerning 656 sows with at least two cycles were studied. The variables used were the following : weight change of the sow before and during reproduction, prolificacy in first cycle, feeding and reproductive rhythm (age of the gilt at mating $212.4 \pm 14.1$; lactation length $27.06 \pm 4.44$; weaning-fertilization interval $6.11 \pm 1.78$, sows being systematically culled when this interval exceeded 14 days). Among these factors, prolificacy in first cycle $(r=0.569)$ was the most involved in the variation of the decrease in prolificacy $(32.3 \mathrm{p} .100)$ ). It was the same for the prolificacy in second cycle, but its effect was positive $(\mathbf{r}=0.187)$. The following factor was the farrowing-mating interval whose lengthening by 10 days improved prolificacy in second cycle by 0.95 piglet. The lactation length and mainly the weaning-fertilization interval had a significant and favourable effect on the prolificacy in second cycle. They did not totally account for the variation in the decrease of prolificacy, but allowed to relate the recent appearance of this phenomenon to the acceleration of reproductive rhythms. The weight variable accounting the best for the effect of the prolificacy in first cycle was the weight loss during lactation. It was not demonstrated any important and significant effect for the dietary factors and the other variables of the weight change.

\section{Sow management according to the three-week batch system}

\author{
M. LE DENMAT *, J. DAGORN *, Françoise DUFOUR * * \\ *Institut Technique du Porc, B.P. 3, 35650 Le Rheu \\ **: Institut Technique du Porc, 149, rue de Bercy, 75595 Paris Cedex 12
}

France

The purpose of the present study was to analyse sow management in 268 farms using the three-week batch system. These farms were selected among the 8000 ones recorded by the "National computerized programme for analysis of on-the-farm sow records ». Additional data were also obtained through an inquiry into the utilization of the farrowing houses. A total of 84.7 p. 100 of matings were synchronized the same week and 73.5 p. 100 of the farrowings. In both cases synchronization of the young females at their first litter was not satisfactory as compared to the other litters. Losses at birth varied in the same way as prolificacy. They were larger in the first sows of the batch to farrow, i.e. those having the shortest time to get accustomed to the farrowing house. Most of the farmers practised weaning on thursday (70.4 p. 100 of the litters), thus matings often took place the next monday, tuesday or wednesday.

With regard to the number of batches or to the number of litters produced during the considered period, the stocking rate was mostly lower than the stocking capacity, especially when there was no extra house available. The production objectives $(93.6 \mathrm{p} .100$ on an average) as compared to the size of the farrowing houses were better in the group with an extra room $(96.5$ p. 100$)$, the latter being used at 48.5 p. 100. 